\title{
Can acute resistance exercise facilitate episodic memory encoding?
}

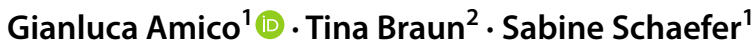

Accepted: 28 September 2021

(c) The Author(s) 2021

\begin{abstract}
Research has shown benefits of physical exercise on memory performance when carried out before or after a memory task. The effects of concurrent physical exercise and particularly resistance exercise are still inconclusive. The current study investigates the influence of resistance exercise with two intensities (fast and slow squats) on performance in a wordlist learning task using a within-subject design. Sport students $\left(N=58, M_{\text {age }}=23\right.$ years; 26 women $)$ were trained in a mnemonic technique to encode word lists (method of loci). In each session they were asked to encode two lists, each consisting of 20 words. During encoding, participants either performed one squat per word (fast-squat-condition), one squat every second word (slow-squat-condition), or stayed seated (control-condition). Participants performed three sessions for each condition, in counterbalanced order. Heart rates differed significantly according to exercise intensity. Memory performances in the sitting condition were better, compared to the exercise conditions. Performance in sitting and the fast squat conditions improved similarly over time, while performance in the slow squat condition increased faster, and reached the level of the fast squat condition at the end of the study phase. We conclude that light to moderate resistance exercise while working on an episodic memory task may rather represent a dual-task situation (= two tasks that compete for attentional resources). Especially doing a squat every second word may represent an inhibition task that people have to get used to. Future studies should include biochemical markers of arousal and neuronal plasticity in addition to heart rate.
\end{abstract}

Keywords Episodic memory $\cdot$ Acute physical exercise $\cdot$ Resistance exercise $\cdot$ Method of loci

\section{Introduction}

Cognition is an essential ability needed to learn, act and remember. Thus, it is important to investigate possible ways that can enhance cognitive functions. Chronic physical exercise has been shown to improve cognition (Hillman et al., 2011; Singh et al., 2018; Tomporowski et al., 2008). Furthermore, an acute bout of physical exercise can be beneficial for various cognitive functions (Chang et al., 2012b; Verburgh et al, 2014), including memory (Loprinzi et al., 2019a; McMorris \& Hale, 2012; Roig et al., 2013, 2016; Tomporowski, 2003).

Gianluca Amico

gianluca.amico@uni-saarland.de

1 Department of Sport Sciences, Institute for Sport Science, Saarland University, Saarland University, Campus Building B 8.1, 66123 Saarbrücken, Germany

2 Department of Psychology, Institute of Psychology, University of the Bundeswehr Munich, 85577 Neubiberg, Germany
The neuropsychological and neurophysiological mechanisms that underlie these positive effects of physical exercise on cognition have not been thoroughly investigated. Factors that are affected by physical exercise are changes in physiological arousal, the release of neurotransmitters, and nerve growth factors (Loprinzi \& Frith, 2019; Loprinzi et al., 2017, 2018a; McMorris, 2016).

Physical exercise improves physiological arousal, including alertness, resource allocation, and executive control, presumably by increasing neuronal excitability (Audiffren et al., 2008; Loprinzi et al., 2018a; McMorris \& Graydon, 2000). These changes in physiological arousal can be linked to changes in heart rate, blood pressure, and skin conductivity, which are closely associated with changes in the concentration of various endogenous neurotransmitters (Cooper, 1973; McMorris, 2016). One of these transmitters is norepinephrine (NE), which controls the activation of the noradrenergic system. This is a possible pathway to improve LTP via activation of $\beta$-receptors (Hansen \& Manahan-Vaughan, 2015; Loprinzi et al., 2018a). In this context, Segal et al. (2012) showed that exercise significantly 
increased NE concentration, and was positively correlated with memory performance. In addition, a study by Cahill and Alkire (2003) demonstrated that an intravenous injection of NE after memory encoding improved the memory consolidation process. Dopamine (DA), serotonin (5-HT), and acetylcholine (ACh) seem to have a mediating effect on hippocampal CA1 structure and thus facilitate LTP (Loprinzi et al., 2018a). DA and NE closely interact with each other. While DA regulates the filtering of signals, NE increases the response to incoming signals (Finlay et al., 1995). 5-HT, in turn, activates the dopaminergic system via receptors in the medial prefrontal cortex (mPFC) (Díaz-Mataix et al., 2005) and may also facilitate NE release (McMorris, 2016).

The level of various nerve growth factors like brain derived neurotropic factor (BDNF), vascular endothelial growth factor (IGF-1) and neurotransmitters (like epinephrine (EPI), NE or DA) can be elevated by physical exercise. These substances are positively associated with memory and learning (Skriver et al, 2014). Nerve growth factors and catecholamines may also influence episodic memory. In this context, Winter et al. (2007) investigated BDNF, catecholamine concentrations (EPI, NE, DA), and memory performance in a word-picture pseudoword learning task, after a moderate endurance exercise, after intense sprint exercise, or after a resting period. The intense exercise group showed the highest increases in BDNF and catecholamine concentrations, and was faster in learning but not in the retention tests. Increased BDNF was associated with better memory performance immediately after learning, while DA concentration correlated with performance after 1 week and EPI with performance after 8 months. These results show that the influence of neurobiological markers is time-dependent and may affect different steps of the memory process, namely memory encoding, memory consolidation, and memory retrieval (Loprinzi et al., 2017, 2019b). The time dependency could be due to different time courses for exerciseinduced increases in various neurosubstrates and their metabolic breakdown (see Meeusen \& Meirleir, 1995 for DA, $\mathrm{NA}$, and 5-HT in rat striatum).

In the past, positive effects of acute exercise bouts on memory have been observed for aerobic exercises, and for resistance exercises. These two types of studies are summarized in the following sections.

\section{Effects of Acute Aerobic Exercise on Episodic Memory}

The current study focused particularly on how physical exercise can affect the processes underlying episodic memory. Most studies so far have focused on exercise bouts before or after memory encoding (Coles \& Tomporowski, 2008; Etnier et al., 2014; Hötting et al., 2016; Labban \& Etnier, 2011; Pesce et al., 2009; Pyke et al., 2020; Schramke \&
Bauer, 1997). Acute exercise during memory encoding has hardly been investigated (Loprinzi et al., 2019a), but it may have a great potential for improving episodic memory. Exceptions are three studies by Maren Schmidt-Kassow and colleagues (Schmidt-Kassow et al., 2010, Schmidt-Kassow et al., 2013, Schmidt-Kassow et al., 2014). For all studies, participants learned word pairs from their native language (German) and from languages that they did not know well (French or Polish).

Schmidt-Kassow et al. (2010) had participants learn the word pairs either while cycling at moderate intensity, or while sitting. Recall of the corresponding German words was assessed after every third learning session. Participants of the cycling group showed higher performance in the vocabulary test compared to the sitting group. In addition, the authors measured a larger N 400 effect in event-related potentials (ERP) in the physically active group, indicating exercise-induced changes in cortical plasticity.

There are two effects described in the literature that could be related to the observed memory benefits: "synaptic tagging" and the "associativity effect" (Frey \& Morris, 1997; Li et al., 2014; Sajikumar \& Frey, 2004). Both effects describe the co-occurrence of a weak (e.g. vocabulary) and a strong input (exercise). Exercise can induce a high action potential (associativity) or trigger plasticity-related proteins that are captured by the memory stimulus (synaptic tagging), increasing LTP of the memory stimulus (Loprinzi et al., 2018a).

In a later study, Schmidt-Kassow et al. (2013) asked participants to learn word-pairs in different exercise conditions. While one group bicycled before learning, one bicycled during learning, and a third group did not exercise. Again, the memory performance of the group that exercised during learning was improved compared to the sedentary group. Elevated heart rates during exercise and increased releases of memory enhancing neurotransmitters may have optimized the physiological arousal during the encoding process, facilitating LTP. However, the authors did not find any correlations of BDNF in serum, or of BDNF genotype, with memory performance. This may be explained by the low intensity of the exercise, as earlier studies have shown that BDNF increases with rising exercise intensity (Hötting et al., 2016; Knaepen et al., 2010; Winter et al., 2007).

In another study, Schmidt-Kassow et al. (2014) tested young adults in a within-subject design. In one session, participants learned word pairs while walking at preferred speed, and in the other session while sitting. Results showed that even a low-intensity activity like walking can improve memory performance compared to no exercise. The muscle spindle pathway described by Loprinzi et al. (2018a) may offer a possible explanation. In particular, walking leads to the contraction of various muscles of the leg. These contracting muscle spindles may create action potentials that are 
transmitted from the spinal cord to the brain stem and from there to the nucleus tractus solitarii (NTS). The activation of the NTS then stimulates the production of neurotransmitters in the locus coeruleus (LC), which has connections to multiple hippocampal structures, that may enhance the memory process (Loprinzi et al., 2018a).

\section{Effects of Acute Resistance Exercise on Episodic Memory}

The studies by Schmidt-Kassow and colleagues used aerobic exercises like walking and cycling, with exercise intensities that were low to moderate. So far, the literature has mainly focused on cardiovascular exercise, but there is a growing interest in positive effects of acute resistance exercise on cognition (Chang \& Etnier, 2009a, 2009b; Chang et al., 2012a; Harveson et al., 2016; Landrigan et al., 2019; Wilke et al., 2019), and particularly on memory (Cuttler et al., 2018; Weinberg et al., 2014). In addition, both types of exercise affect memory through distinct molecular pathways, indicating that aerobic exercise modulates the BDNF and $\beta$-CaMKII (calcium/calmodulin-dependent kinase II) while resistance training influences the IGF-1 and AKT (protein kinase B) pathway (Cassilhas et al., 2012).

In this context, Weinberg et al. (2014) tested 46 young adults in an emotional episodic memory task in a betweensubject design. On day 1, participants encoded 90 images (30 negative, 30 positive, 30 neutral). Directly after memory encoding, they either performed 2 sets of a one-leg knee extension/flexion exercise (exercise group) or remained seated while their leg was passively moved by the experimenter (control group). On day 2, participants had to recognize the previously seen images while 90 new images were added to the recall task. To assess the physiological reaction to the exercise, salivary alpha amylase (a biomarker of norepinephrine), heart rate, and blood pressure were measured. Results showed higher accuracy rates for the exercise group in the recognition task. Furthermore, within the exercise group, participants that showed a high physiological response to the exercise had a lower performance in recognizing neutral images, but not in recognizing positive or negative pictures, compared to participants with a lower physiological response to the exercise. The authors conclude that an acute resistance exercise can facilitate episodic memory and may be particularly beneficial for emotional memory. As far as we know, the current study will be the first to investigate the effects of a concurrent resistance exercise on episodic memory performance.

\section{Hypotheses of the Current Study}

The current study investigates the effects of resistance exercise on memory encoding in university sports students. Each participant was instructed and trained in the method of loci as a memory strategy (Amico \& Schaefer, 2019). Over the course of a seminar, participants learned two new lists of 20 words per session, while they either remained seated (sitting condition), performed one squat every second word (slowsquat-condition), or one squat every word (fast-squat-condition). Squats are a resistance exercise, comprised of slow, easy, and rhythmic movements. Squats do not create much acoustic noise when performed in a group, reducing distractions as much as possible. Participants performed three sessions for each condition. Based on previous studies showing a positive effect of acute aerobic exercise during memory encoding (Schmidt-Kassow et al., 2010, 2013, 2014) and the study by Weinberg et al. (2014) showing that an acute resistance exercise can benefit episodic memory, we hypothesized that students will remember more words with increasing intensity in the exercise conditions (fast-squat $>$ slowsquat $>$ sitting). Note that exercise intensity remains between a low and medium level in the current study. This hypothesis is further grounded on the acute effects of physical exercise on LTP (Loprinzi et al., 2017, 2018a; McMorris, 2016) and by optimizing neuronal excitability and resource allocation (Audiffren et al., 2008; Loprinzi et al., 2018a; McMorris \& Graydon, 2000). We measured heart rates throughout the testing phase as an indicator of exercise-induced arousal. Lastly, we predicted that participants would show performance improvements with practice of the MoL task over the course of the study. For exploratory reasons, we also assessed whether the expected benefits occur when three lists (60 words) are tested in immediate succession in the last session of the study (e.g., testing-the-limits paradigm by Kliegl et al., 1989). The current study has been preregistered under the following link: https://aspredicted.org/blind. php? $=$ wi2dk7.

\section{Methods and Materials}

\section{Participants}

Fifty-eight sport students from the Saarland University participated in the study in exchange for course credit (see Table 1 for descriptives). All participants had normal or corrected-to-normal vision and hearing and gave informed consent to the study. Participants enrolled in one of three seminar groups, in which the same content was taught. The seminar sessions took place on different days. Fifteen participants enrolled in the Monday seminar group, $n=13$ in the Wednesday seminar group, and $n=30$ in the Thursday seminar group. Please note that it is unlikely that the selection of a specific seminar group is related in any meaningful way to cognitive performances, and that the only difference between groups was the order of experimental conditions 
Table 1 Descriptive statistics of the variables

\begin{tabular}{|c|c|}
\hline Characteristic & $\begin{array}{l}\text { Participants } \\
(N=58)\end{array}$ \\
\hline Age in years $(S D)$ & $M=23.05(2.11)$ \\
\hline Gender $(n)$ & 26 women, 32 men \\
\hline Digit Symbol $(S D)$ & $M=63.69(11.39)$ \\
\hline \multicolumn{2}{|l|}{ Fitness variables } \\
\hline Max. Squats pretest [\%] & 86.3 reached max score \\
\hline Max. Squats posttest [\%] & 73.2 reached max score \\
\hline $\mathrm{HR}$ max. Squats pretest $\left[\% \mathrm{HR}_{\max }\right](S D)$ & $M=72.67(9.27)$ \\
\hline $\mathrm{HR}$ max. Squats posttest $\left[\% \mathrm{HR}_{\max }\right](S D)$ & $M=64.54(10.13)$ \\
\hline \multicolumn{2}{|l|}{ MoL task variables } \\
\hline Location cues [\%] & 77.6 reached max score \\
\hline \multicolumn{2}{|l|}{ Heart rate $\left[\% \mathrm{HR}_{\max }\right]$} \\
\hline Sitting $(S D)$ & $M=41.40(5.56)$ \\
\hline Slow squats $(S D)$ & $M=52.97(6.82)$ \\
\hline Fast squats $(S D)$ & $M=57.78(7.01)$ \\
\hline \multicolumn{2}{|l|}{ Memory score } \\
\hline Sitting $(S D)$ & $M=13.53(4.39)$ \\
\hline Slow squats $(S D)$ & $M=10.27(4.13)$ \\
\hline Fast squats $(S D)$ & $M=12.12(4.29)$ \\
\hline
\end{tabular}

The $n$ may differ slightly for some variables due to missing data

over the course of the study (see Procedure section). To have similar samples sizes across groups, the Monday and Wednesday groups were combined before data collection started. The study was approved by the Ethics committee of Saarland University.

\section{Method of Loci Task}

The Method of Loci task (MoL) is a well-established memory strategy to encode word lists (Amico \& Schaefer, 2019; Kliegl et al., 1990; Li et al., 2001; Schaefer et al., 2008). To start with, participants learned a sequence of location cues. In the current study, the cues corresponded to 20 locations that are part of every apartment (e.g., bed, window, table, chair). The to-be-learned words were taken from Brehmer et al. (2004) and consisted of concrete German nouns that can be easily imagined, like objects, animals or professions. Participants heard lists of 20 words, read out by the experimenter with an inter-stimulus-interval of $2.5 \mathrm{~s}$. The instruction was to encode the to-be-learned word by combining it with the respective location cue via mental imagery. Participants were encouraged to include object size, sound, touch, emotions, or movement depending on their personal preferences. For example, when encoding the word "spider" at the location "table", a participant could imagine a huge hairy spider crawling over the table. During encoding and recall, participants did not have access to the list of locations. Immediately after the last word was presented, participants wrote down the remembered words in the correct order on their answer sheets. There was no time limit for recall. At the start of each seminar session, two lists were administered except for the first two sessions, in which only one list was presented. Altogether, $28 \mathrm{MoL}$ lists had been constructed and were distributed randomly across the experimental sessions for each seminar group, to control for potential differences in the task-difficulty of individual lists. The dependent variable for MoL was the sum of correctly remembered words at the correct location. To encourage learning of the location cues, participants were asked to reconstruct the list of 20 locations at the beginning of each session, by writing them down on a sheet of paper. To sustain motivation over the course of the semester, the ten candidates of each seminar who scored highest in the $\mathrm{MoL}$ task received monetary rewards (Place 1: 15 Euro, Place 2: 10 Euro, Place 3 to 10: 5 Euro) at the end of the semester.

In order to investigate the effect of acute resistance exercises on episodic memory performance, participants either performed one squat for every word presented (fast squat condition), one squat for every second word presented (slow squat condition), or stayed seated during memory encoding (sitting condition). The order of the exercise conditions was counterbalanced across the groups. In the squat conditions, participants began to do squats $9 \mathrm{~s}$ before the lists started, to get a feeling for the rhythm of the respective condition. An auditory signal indicated when a squat had to be performed. Please note that the distribution of participants across the room was the same as for the maximum performance squat task (see below). After presentation of the to-be-learned words, participants stopped the exercise and recalled the words by writing them down.

\section{Covariates}

Heart Rate Heart rate was measured during the presentation of each word list in the MoL task and during the maximum performance squats test (see below) with hardware and software from Polar Team ${ }^{2}$. The mean heart rate during this time interval was converted from beats per minute [bpm] to percent of maximum heart rate $\left[\% \mathrm{HR}_{\max }\right]$. Maximum heart rate was estimated using the formula 220 - age.

Maximum Performance Squat Task In the first and the last session, all participants performed a "maximum performance" squat test. The test was administered to assess potential individual performance limitations in the squats task, and to estimate how physically demanding the squats are. During the squats, participants stood with their feet approximately shoulder-width apart. Participants were spaced equally across the room with a distance of roughly one meter between them, to further limit possible distractions. Participants were also not facing each other, to avoid non-verbal 
communication. Their feet were externally rotated about $0^{\circ}$ to $5^{\circ}$ degrees, and knees were aligned over the tip of the toes. The instruction was to flex the knee between $100^{\circ}$ to $130^{\circ}$ degrees (see Fig. 1). Typical errors of movement execution were corrected in the first session (curved back, excessive forward lean of the torso, looking at the ground, etc.). The squats were performed in the rhythm of the fast-squat condition (one squat every $2.5 \mathrm{~s}$ ). For the maximum performance squat task, the upper limit of squats that could be reached was 256 . Participants were instructed to do as many squats as possible, either until exhaustion forces them to stop, or until the end of the test was reached. Heart rates were assessed throughout the test. The vast majority of participants reached the maximum possible score, therefore the maximum performance squats was assessed dichotomously with 1 indicating the maximum number of squats was reached (see Table 1).

Location Cues Before the MoL Task, each participant had to recall the list of the 20 location cues for the MoL task, by writing them down on a sheet of paper. Each correct location scored 1 point. Participants were instructed to use the same locations in every trial of the MoL task. The overall performance in retrieving the location cues was very high, therefore we assessed the location cues dichotomously with 1 indicating maximum score was reached (see Table 1).

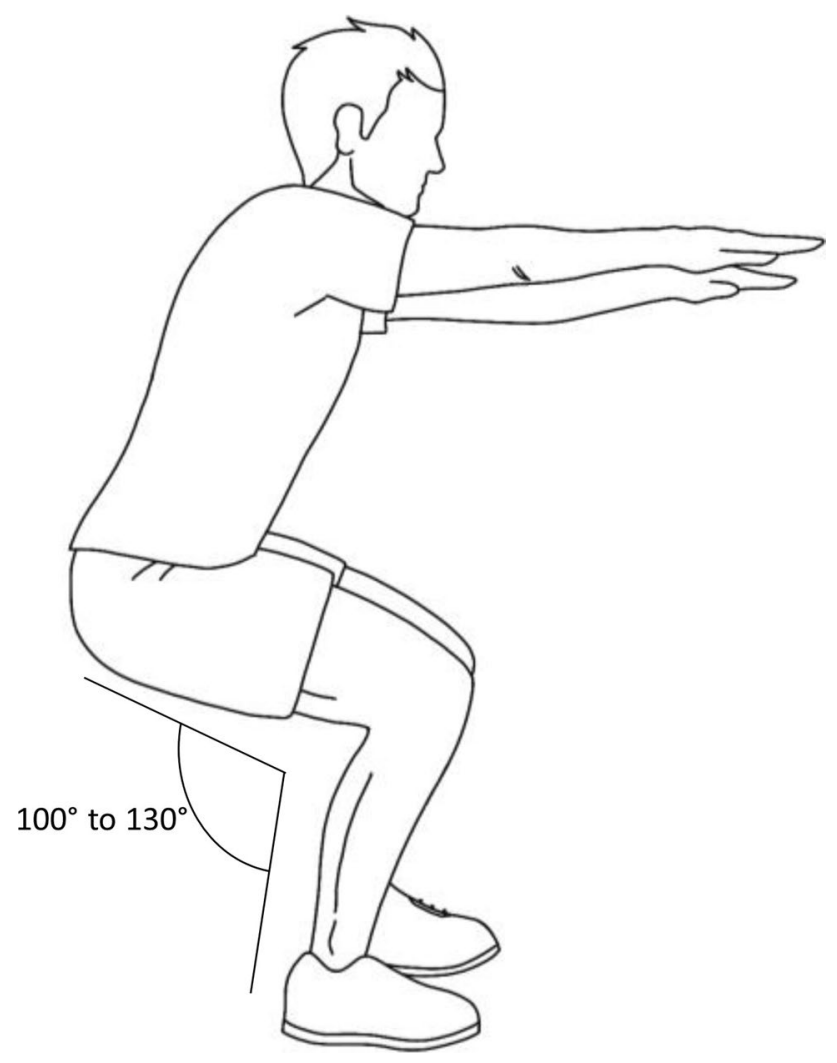

Fig. 1 Schematic illustration of the performance of a squat
Digit Symbol Substitution Test Perceptual speed was measured with the Digit-Symbol Substitution task (Wechsler, 1981) as a covariate in the main analysis, and as a background variable. For this paper-and-pencil-test, participants fill in blank spaces underneath numbers with the corresponding abstract symbol as quickly as possible. Scores can range between zero and 93. An ANOVA with seminar group as between-subjects factor showed no differences in perceptual speed across the three groups, $F(2,55)=0.066, p=0.936$. In addition, Digit Symbol scores corresponded well to young adult samples of other representative studies (see Amico \& Schaefer, 2019) (see Table 1).

\section{Procedure}

The study consisted of 3 preparation sessions and 12 testing sessions for each group, with one session per week per group. Table 2 presents an overview of the testing regime. The first three sessions form the preparation phase. In the first preparation session, participants completed a demographic questionnaire, the Digit-Symbol Substitution test and one trial of a memory task that was similar to the MoL task explained above, except that no instructions of any memory strategy and no location cues were given. In the second preparation session, MoL was explained as a memory strategy and the importance of individual preferences for specific aspects of the strategy (e.g., size, movement, emotions) was emphasized. Then the list with 20 locations was presented and the participants were asked to mentally

Table 2 Experimental procedure by seminar group and session

\begin{tabular}{lll}
\hline Session number & Group 1 & Group 2 \\
\hline Preparation 1 & Demographics & Demographics \\
Preparation 2 & Instruction & Instruction \\
Preparation 3 & MoL familiarization & MoL familiarization \\
1 & Baseline Pretest, & Baseline Pretest, \\
& Max-Performance & Max-Performance Squats \\
& Squats & \\
2 & Sitting & Fast squats \\
3 & Slow squats & Slow squats \\
4 & Fast squats & Sitting \\
5 & Sitting & Fast squats \\
6 & Slow squats & Slow squats \\
7 & Fast squats & Sitting \\
8 & Sitting & Fast squats \\
9 & Slow squats & Slow squats \\
10 & Fast squats & Sitting \\
11 & Baseline Posttest, & Baseline Posttest, \\
& Max-Performance & Max-Performance Squats \\
& Squats & \\
12 & Testing the limits (sit- & Testing the limits (fast \\
& ting) & squats) \\
\hline
\end{tabular}


visualize these places in their home. MoL was trained with one trial, by constructing examples of mental images with the help of the experimenter. In the third preparation session, participants were asked to draw a sketch of their apartment and to mentalize the location cues. After that, the Mol task was practiced with two more lists to familiarize participants with the task. Session 1 provided the data for the baseline pretest assessment of the MoL task. Then the maximum performance squat test was carried out. In each of the following 9 sessions, participants completed two trials of the MoL task, with one out of the three conditions described above (sitting, slow squats, fast squats). The order of these conditions was counterbalanced, using a $2 \times 3$ Latin square design (see Table 2). In session 11, participants completed two trials of the MoL task without any additional task (baseline posttest), and a second trial of the maximum performance squats task. In session 12, the MoL task was performed with three lists being presented nonstop (altogether 60 words), as an exploratory testing-the-limits situation. Group 1 stayed seated and group 2 performed fast squats. The lists used were newly constructed lists of words that had already been tested previously. Session 12 enabled us to compare the effect of an acute physical resistance exercise to a non-exercising group for a particularly challenging memory task.

\section{Analyses}

The data displayed a hierarchical data structure with measurements (Level 1) being nested in individuals (Level 2). Accordingly, we applied a multilevel approach (Raudenbush \& Bryk, 2002; Snijders \& Bosker, 2004), which allows us to compare means and investigate associations while controlling for the dependencies in the dataset. We conducted a step-wise multilevel analysis to test our hypotheses. Our first research question investigated whether participants achieve higher scores in the MoL task during the exercise conditions, namely slow squats and fast squats, compared to the sitting condition. The second research question tested whether the heart rate during memory encoding is associated with scores in the MoL task. Thirdly, we predicted that performance in the MoL task would increase over the course of the seminar. To this end, in Model 1.1 the score in the memory task was regressed on the dummy coded condition and grandmean centered heart rate. The analysis was controlled for the number of the sessions, grand-mean centered percent of maximum heart rate $\left[\% \mathrm{HR}_{\max }\right]$ when testing maximum performance squats in the pretest and score in the Digit Symbol Substitution test, as well as dichotomous number of squats in the maximum performance squat pretest and score on the list of locations cues. In a second step, an interaction between number of sessions and condition was added to the model, in order to explore whether participants improved differently over time within the different conditions (Model 1.2).
As a manipulation check, heart rate during the MoL task was also regressed on number of sessions and dummy coded condition (Model 2). This allowed to test whether the different conditions lead to different heart rate levels as assumed. An interaction of heart rate and condition did not improve the model and was therefore not further analyzed. A final follow-up analysis explored whether the conditions would also differ in the testing the limits session. Therefore, the score on the testing the limits test was regressed on dummy coded number of list ( 1 vs. 2 vs. 3 ) and dichotomous condition (sitting vs. fast squat, Model 3).

All analyses were conducted using lme4 (Bates et al., 2015) and lmerTest (Kuznetsova et al., 2015), packages for multilevel analysis in R.

\section{Results}

\section{Descriptive Analyses of the Fitness Variables}

To describe the participants' fitness level and the exercise intensities of the MoL task conditions, descriptive statistics are presented below.

\section{Maximum performance squats}

The vast majority of participants of the current study were able to perform 256 squats in a row (see Table 1). 44 out of 51 participants finished all 256 squats in the pretest, and 30 out of 41 participants finished all 256 squats in the posttest. The few participants who did not succeed in finishing all squats performed at least 111 squats in the pretest or 75 squats in the posttest (lowest performances observed throughout all participants). This indicates that performing 20 squats in a row while encoding the word lists (or 60 squats in the testing-the-limits session) was not a physical challenge to our participants.

\section{Heart rate during the maximum performance squats and the MoL task}

As expected, the heart rates $\left[\% \mathrm{HR}_{\max }\right]$ during the maximum performance squat pre- and posttest are far from being an intensive cardiovascular strain for this age group (Physical Activity Guidelines Advisory Committee, 2008). Although heart rates seem to decrease from pre- to posttest, this does not indicate training effects, but rather represents a correlation with the higher number of participants who stopped the maximum performance squat posttest compared to the pretest (see Table 1). In addition, the heart rates during the exercise conditions (slow squats, fast squats) show that heart rates were comparable to other studies testing acute effects 
of exercise during memory encoding (Schmidt-Kassow et al., 2010, 2014).

\section{MoL Task Performance}

To test the amount of variance proportions at both levels of analyses (between measurements and between individuals), we estimated an unconditional model of MoL task performance, to examine the distribution of between-person and within-person variation. An intraclass correlation of 0.48 for MoL task performance suggests that roughly 50 percent of the total variation of MoL task performance can be explained by the between-person portion of total variation. Thus, a substantial amount of variation existed between session numbers (Level 1) and between individuals (Level 2).

Results of the first set of multilevel analyses are summarized in Table 3. As predicted, participant's performance in the MoL task increased over the course of the sessions (see Model 1.1). However, contrary to the predictions of our first hypothesis, memory performance in the MoL task was better in the sitting condition compared to the slowand the fast-squat condition. In addition, performance in the fast-squat condition was better compared to the slow-squat condition (Est. $=-2.01, S E=0.31, p<0.001)$. Contrary to our second hypothesis, heart rate during the MoL task did not significantly affect MoL task performance. Regarding the measured covariates, only the Digit Symbol test partially explained performance in the MoL task, indicating that higher performance in the Digit Symbol task is associated with higher performance in the MoL task. As an exploratory analysis, an interaction between number of sessions and condition was added to the model (Model 1.2). The MoL performance in all three conditions increased over the course of the sessions. In addition, the performance in the sitting and the fast squat condition increased parallel over time while the performance in the slow squat condition showed a steeper increase compared to both other conditions (see Fig. 2 for the pattern of results).

\section{Manipulation Check: Heart RATE DURING the MoL Task}

To test the amount of variance proportions at both levels of analyses (between measurements and between individuals), we estimated an unconditional model of the heart rate during the MoL task, to examine the distribution of
Table 3 Score in the MoL task by condition and heart rate during MoL

\begin{tabular}{|c|c|c|c|c|c|c|}
\hline & \multirow{2}{*}{\multicolumn{2}{|c|}{$\frac{\text { Model } 1.1}{\text { Ref: Sitting }}$}} & \multicolumn{4}{|c|}{ Model 1.2} \\
\hline & & & \multicolumn{2}{|c|}{ Ref.: Sitting } & \multicolumn{2}{|c|}{ Ref.: Fast squats } \\
\hline & Est & SE & Est & SE & Est & SE \\
\hline \multicolumn{7}{|l|}{ Fixed effects } \\
\hline Intercept & $9.92 * *$ & 1.13 & $10.13 * *$ & 1.12 & $8.39 * *$ & 1.24 \\
\hline Session & $0.43 * *$ & 0.05 & $0.32 * *$ & 0.06 & $0.36^{* *}$ & 0.09 \\
\hline Heart rate during MoL & -0.24 & 0.25 & -0.24 & 0.25 & -0.24 & 0.25 \\
\hline \multicolumn{7}{|l|}{ Condition } \\
\hline Sitting vs Fast squat & $-1.51 *$ & 0.48 & $-1.74 *$ & 0.74 & $1.74 *$ & 0.74 \\
\hline Sitting vs Slow squat & $-3.53 * *$ & 0.41 & $-6.68 * *$ & 0.68 & & \\
\hline Fast vs Slow squat & & & & & $-4.93 * *$ & 0.75 \\
\hline \multicolumn{7}{|l|}{ Condition X Session } \\
\hline Sitting vs Fast squat & & & 0.04 & 0.09 & -0.04 & 0.09 \\
\hline Sitting vs Slow squat & & & $0.54 * *$ & 0.09 & & \\
\hline Fast vs Slow squat & & & & & $0.50 * *$ & 0.12 \\
\hline Digit Symbol & $1.45^{*}$ & 0.52 & $1.41 *$ & 0.52 & $1.41 *$ & 0.52 \\
\hline Max Squats $(1=\max$ reached $)$ & 1.10 & 1.07 & 1.06 & 1.06 & 1.06 & 1.06 \\
\hline HR during max squats & 0.37 & 0.52 & 0.35 & 0.51 & 0.35 & 0.51 \\
\hline Location cues $(1=$ all correct $)$ & 0.76 & 0.79 & 1.21 & 0.78 & 1.21 & 0.78 \\
\hline Random effects & Var & $S D$ & Var & $S D$ & Var & $S D$ \\
\hline Intercept & 13.95 & 3.74 & 13.87 & 3.72 & 13.87 & 3.72 \\
\hline Session & 0.05 & 0.23 & 0.05 & 0.23 & 0.05 & 0.23 \\
\hline Residual & 10.44 & 3.23 & 10.00 & 3.16 & 10.00 & 3.16 \\
\hline \multicolumn{7}{|l|}{ Model fit } \\
\hline AIC & 4713.6 & 4684.1 & 4684.1 & & & \\
\hline
\end{tabular}

The models are two-level models with 872 observations on level 1 and 47 individuals on level 2. ** $p<0.001 ; * p<0.05$ 
Fig. 2 Results of the MoL task for the three conditions, sitting, fast squats, and slow squats over the course of the seminar sessions. Note. Every participant took part in every condition. The order of conditions across sessions is shown in Table 2

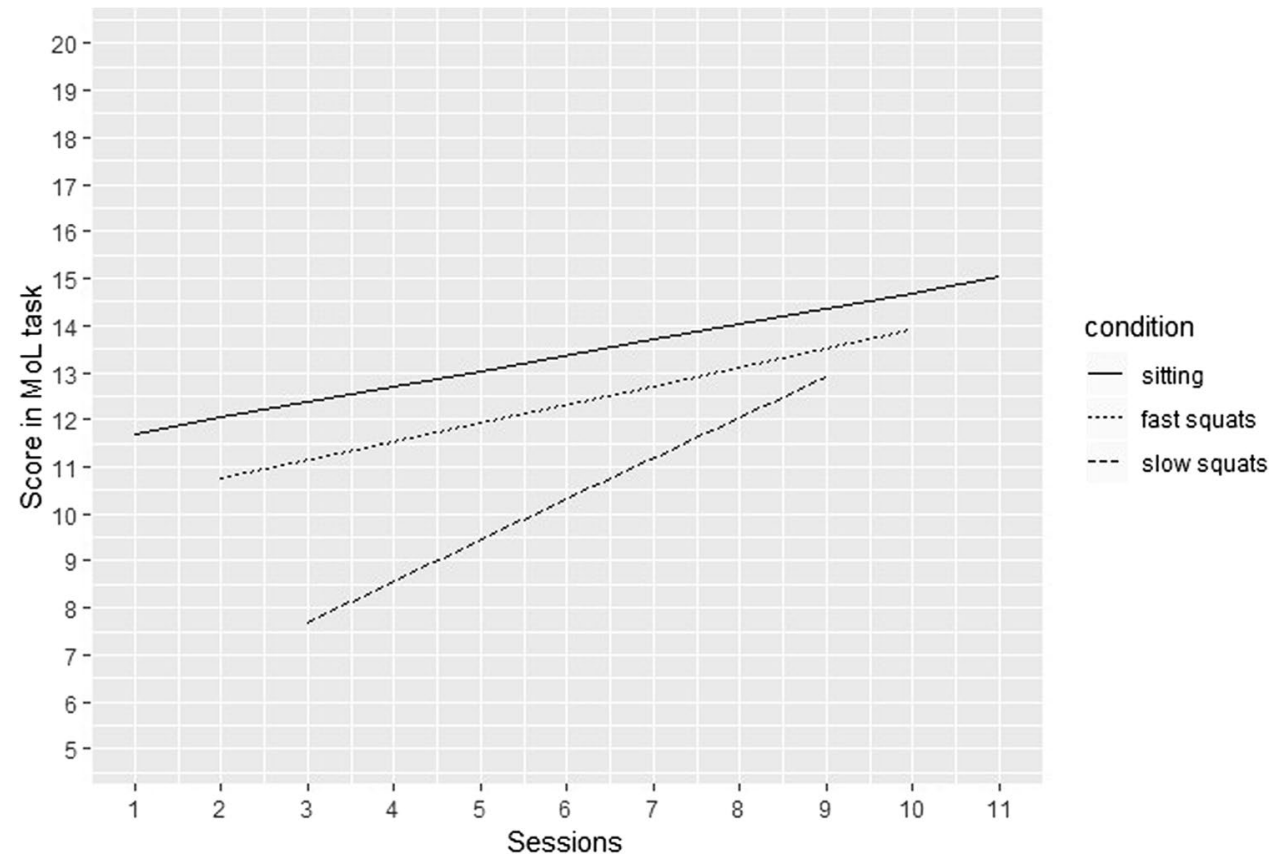

Table 4 Heart rate during the MoL task by condition

\begin{tabular}{|c|c|c|c|c|}
\hline & \multicolumn{4}{|l|}{ Model 2} \\
\hline & \multicolumn{2}{|c|}{ Ref.: Sitting } & \multicolumn{2}{|c|}{ Ref.: Fast squat } \\
\hline & Est & SE & Est & SE \\
\hline \multicolumn{5}{|l|}{ Fixed effects } \\
\hline Intercept & $2.40 * *$ & 0.04 & $1.70 * *$ & 0.04 \\
\hline Session & $0.01 * *$ & 0.00 & $0.01 * *$ & 0.00 \\
\hline \multicolumn{5}{|l|}{ Condition } \\
\hline Sitting vs Fast squat & $-0.70 * *$ & 0.02 & $0.70 * *$ & 0.02 \\
\hline Sitting vs Slow squat & $-0.54 * *$ & 0.01 & & \\
\hline Fast vs Slow squat & & & $0.16^{* *}$ & 0.02 \\
\hline Random effects & Var & $S D$ & Var & $S D$ \\
\hline Intercept & 0.06 & 0.25 & 0.06 & 0.25 \\
\hline Session & 0.00 & 0.02 & 0.00 & 0.02 \\
\hline Residual & 0.04 & 0.19 & 0.04 & 0.19 \\
\hline \multicolumn{5}{|l|}{ Model fit } \\
\hline AIC & -213 & -213 & & \\
\hline
\end{tabular}

The models are two-level models with 1027 observations on level 1 and 55 individuals on level 2. ** $p<0.001 ; * p<0.05$

between-person and within-person variation. An intraclass correlation of 0.29 for heart rate during MoL task suggests that roughly one third of the total variation of the heart rate can be explained by the between-person portion of total variation. Therefore, a substantial amount of variation lies between session numbers (Level 1) and between individuals (Level 2).

Results of the second set of multilevel analyses are summarized in Table 4 (Model 2). As predicted, participant's heart rate differed significantly between all the three conditions, indicating that participant's cardiovascular activity was highest during fast squats, second highest during slow squats, and lowest during the sitting condition. Although there was a statistically significant change in heart rate over the sessions (decrease in heart rate of $0.011 \%$ per session), this result has no further practical implication for the pattern of results, as it describes an overall change in heart rate of roughly $1 \%$ over the total eleven sessions.

\section{Exploratory Analysis: Testing the Limits Session}

To test the amount of variance proportions at both levels of analyses (between measurements and between individuals), we estimated an unconditional model of the memory score in the testing the limits session, to examine the distribution of between-person and within-person variation. An intraclass correlation of 0.22 for memory performance suggests that roughly one fifth of the total variation of memory performance can be explained by the betweenperson portion of total variation.

Results of the multilevel analysis are summarized in Table 5 (Model 3). Results show the best recall performance for list 1 , followed by list 3 , and worst performance for words of list 2, indicating primacy and recency effects. Contrary to our predictions, memory performance in the sitting condition was better compared to the fast squat condition in the testing the limits session. 
Table 5 Score in the testing the limits session by list number and condition

\begin{tabular}{|c|c|c|c|c|}
\hline & \multicolumn{4}{|c|}{ Model 3} \\
\hline & \multicolumn{2}{|c|}{ Ref.: list 2} & \multicolumn{2}{|c|}{ Ref.: list 1} \\
\hline & Est & SE & Est & SE \\
\hline \multicolumn{5}{|l|}{ Fixed effects } \\
\hline Intercept & $4.29 * *$ & 0.79 & $8.68 * *$ & 0.79 \\
\hline \multicolumn{5}{|l|}{ List number } \\
\hline list 1 vs list 2 & $4.39 * *$ & 0.76 & $-4.39 * *$ & 0.76 \\
\hline list 1 vs list 3 & & & $-2.41^{*}$ & 0.76 \\
\hline list 2 vs list 3 & $1.98^{*}$ & 0.76 & & \\
\hline \multicolumn{5}{|l|}{ Condition } \\
\hline Sitting vs Fast squat & $-2.40^{*}$ & 0.93 & $-2.40^{*}$ & 0.93 \\
\hline Random effects & Var & $S D$ & Var & $S D$ \\
\hline Intercept & 4.97 & 2.23 & 4.97 & 2.23 \\
\hline Residual & 11.81 & 3.44 & 11.81 & 3.44 \\
\hline \multicolumn{5}{|l|}{ Model fit } \\
\hline AIC & 698.2 & 698.2 & & \\
\hline
\end{tabular}

The model is a two-level model with 123 observations on level 1 and 41 individuals on level 2 . ${ }^{* *} p<0.001 ; * p<0.05$

\section{Discussion}

The current study examined whether acute resistance exercise with low to moderate intensity can enhance memory encoding and thus facilitate the learning of word lists. Measures of heart rate clearly showed that the physical arousal increased with the intensity level of the exercise. However, contrary to our predictions, memory performance was best when participants remained seated during encoding compared to when participants performed squats for every word (fast squat condition) or for every second word (slow squat condition). Memory performance increased over the course of the study, indicating that participants further familiarized with the squat movement and the MoL task. Furthermore, an interaction of time and condition showed that participants' memory performance in the slow squat condition increased faster over the course of the study, although it only reached the performance level of the fast squat condition. Performance differences between the sitting and the fast squat condition persisted in the last testing-the-limits session, which used an even more difficult version of the memory task, with 60 instead of 20 to-be-encoded items.

\section{Reasons for the Exercise-Induced Performance Deteriorations}

Why did the acute resistance exercise impair memory encoding? It is possible that the timing of the exercise played the crucial role. In the current study, participants performed squats while constructing mental images. One squat had to be performed for each to-be-encoded word (fast squats), or for every second to-be-encoded word (slow squats). The need to execute the squat in the correct time window may have created a dual-task situation, drawing attention away from the memory task. Cognitive-motor dual-task situations have often been shown to elicit performance decrements, since resources have to be shared between two domains (Kahneman, 1973; Navon \& Gopher, 1979; Schaefer, 2014; Wickens, 1991). The temporal coupling of the two actions, performing the squat and creating the mental image, was high in the current study. Nevertheless, doing squats in a certain rhythm is not an automatized task, since each squat has to be initiated in the correct time-window. Triggering the physical movement therefore required some attention, and the attentional demands were even higher in the slowsquat condition. We assume that inhibiting the squat for every second word was particularly attention-demanding for our participants. The experimenters could observe this when testing, since there were several participants who performed squats "out of sequence" in the slow squat condition, especially when first encountering this type of task.

Studies that have combined the MoL memory task with a secondary motor task have often found performance decrements in memory performances (Amico \& Schaefer, 2019; Li et al., 2001; Lindenberger et al., 2000; Schaefer et al., 2008). Motor tasks in these studies were continuous, like keeping one's balance on an unstable surface (Schaefer et al., 2008), walking on a narrow track (Li et al., 2001; Lindenberger et al., 2000), or fidgeting, doodling, and drawing (Amico \& Schaefer, 2019). However, in the study by Amico and Schaefer (2019), kneading a stressball while encoding the MoL word lists did not lead to performance decrements in young adults. This indicates that the attentional requirements of the parallel motor task need to be taken into account when searching for exercise-induced performance enhancements in cognition. Future research should include conditions in which participants can perform the motor task in a self-initiated manner.

The differences between the three conditions (sitting, slow squats, fast squats) decreased with increasing practice in the current study. This indicates that participants required some practice to familiarize themselves with the rhythm of the slow squats. Several theories on motor skill learning (Adams, 1971; Gentile, 1972; Fitts \& Posner, 1967; Meinel, 1960) predict that the initial stages of skill acquisition require more cognitive resources/ attention than later stages. Accordingly, once participants had gotten used to the temporal requirements of combining the memory encoding and the slow squat task, their dual-task costs in memory became smaller. Therefore, future studies should invest enough time to familiarize participants with the motor task, especially when participants have less motor experience than sport students. 
The squatting conditions in the current study represent a resistance exercise with a rather low weight, but multiple repetitions. Performing between 20 and 40 squats during a testing session can be seen as a combination of an aerobic and a resistance exercise. Therefore, it is important to interpret the results of the current study in light of previous resistance exercise but also aerobic exercise studies, and to compare both exercise types regarding their effects on episodic memory.

The studies by Schmidt-Kassow and colleagues (SchmidtKassow et al., 2010, Schmidt-Kassow et al., 2013, SchmidtKassow et al., 2014) used preferred-speed treadmill walking or ergometer cycling as acute exercise activities. Their participants were more successful in learning new vocabulary while moving as opposed to sitting. It is possible that the continuous exercises of moderate intensities were better suited than our paradigm to elicit positive effects on memory. A recent study by Amico and Schaefer (2020) neither found performance benefits nor decreases in young adults who were running while encoding vocabulary (as opposed to sitting). However, teenagers profited from the running condition, and outperformed their peers who encoded the words while standing or while dribbling a basketball.

In the current study, all participants were instructed to use the MoL memory strategy. This controls for the use of other mnemonic strategies (like rehearsal or elaboration), and tends to eliminate floor or ceiling effects in list learning paradigms. The MoL requires participants to create mental images and thus involves brain structures that process visual input. Although squats are an easy and slow movement, watching the movement of others may have disturbed focus and attention on the memory task. To counteract this possible influence, future studies should consider using individualized testing sessions instead of group sessions.

\section{The Timing of the Exercise Bout}

It is possible that acute exercise is more favorable when performed as an active break. Then, exercise would not interfere with cognitive resources required for memory encoding. Several studies and reviews conclude that exercise before or after memory encoding is most effective in enhancing memory function (Chang et al., 2012b; Frith et al., 2017; Loprinzi et al., 2019a; Roig et al., 2013). In addition, Roig et al. (2016) argue that acute exercise before memory encoding can affect the encoding and consolidation process while an acute bout of exercise after memory encoding may only affect memory consolidation. More research is needed to determine how the timing of the exercise affects each step of the memory process, and whether there are differences between aerobic and resistance exercises in this respect.

In the current study, we only measured immediate memory performance. We did not assess memory performance after several hours or days. Possibly beneficial effects of exercise would have become visible at a later time, after the memory formation processes is completed (Roig et al., 2013). In this context, studies have shown performance gains when memory was measured $24 \mathrm{~h}$ or 7 days after encoding, but not when measured after $1 \mathrm{~h}$ (Roig et al., 2012; Skriver et al., 2014). However, our results indicate that the encoding process was disturbed by the exercise, making it unlikely that consolidation takes place for information that could not be encoded previously. Nevertheless, assessing memory performances at later points in time is an interesting issue for future research.

\section{Exercise Intensity}

Another subject worth discussing is the exercise intensity. A number of studies that have shown strong effects of acute exercise on memory have used much more intense exercise protocols than we did (Roig et al., 2012; Skriver et al., 2014; Sng et al., 2018; Winter et al., 2007). However, there are also studies that have shown positive effects of low to medium exercise intensities (Schmidt-Kassow et al., 2010, 2013, 2014). We do not expect the chosen exercise intensities to be the reason for the non-beneficial effects found, as we can show that heart rates during slow squats (mean $=53 \%$ $\mathrm{HR}_{\max }$ ) and during fast squats (mean $=58 \%\left(\mathrm{HR}_{\max }\right)$ are well comparable with heart rates measured in other studies that found beneficial effects (Schmidt-Kassow et al., 2010, 2014). Exercise intensities may need to be interpreted differently between aerobic and resistance exercises. However, the maximum performance squat assessments show that our participants were far from performing at their physical limits when doing the squats during encoding. Inadequate execution of the squat technique may reduce the intensity of the exercise and may increase the maximum number of possible repetitions before muscle exhaustion. A closer and individualized monitoring of movement quality should therefore be implemented in future research with this paradigm.

\section{Neuropsychological Mechanisms}

We still do not know much about the acute effects of resistance exercise on memory function. So far most studies have focused on aerobic exercise programs (Loprinzi et al., 2018b). Evidence from animal studies suggests that resistance and aerobic exercise affect memory via distinct molecular pathways. More specifically, aerobic exercise facilitates BDNF related processes, while resistance exercise triggers changes in IGF-1 related processes (Cassilhas et al., 2012). Future studies should further compare both types of exercise and investigate how they affect memory.

Regarding the underlying mechanisms, we expected that physiological arousal would increase to a moderate level 
when doing the squats, which in turn should increase the capacity of the cognitive system. In addition, we assumed that participants' optimized level of arousal would improve resource allocation, enabling the participants to focus on memory encoding (Audiffren et al., 2008; Loprinzi et al., 2018a; McMorris \& Graydon, 2000). Although we found that heart rates increased with rising intensity, there was no systematic relationship between heart rates and memory performance. The current study was limited in measuring other relevant neurobiological markers like BDNF or IGF1 , which would have given more insight into the underlying processes. However, results from earlier studies indicate that BDNF and IGF-1 show larger effects after high intensity exercise (Knaepen et al., 2010; Skriver et al., 2014; Winter et al., 2007), while studies with lower intensity levels did not find correlations between BDNF and memory performance (Schmidt-Kassow et al., 2013, 2014). Interesting candidate substances that may mediate acute exercise effects on memory encoding are the neurotransmitters norepinephrine (NE) and dopamine (DA), due to their characteristic of regulating attention and filtering incoming signals (Finlay et al., 1995). In addition, it would have been interesting to analyze cortisol levels during the MoL task, as research has shown that increased levels of cortisol are associated with improvements in emotional memory (Buchanan \& Lovallo, 2001; Kuhlmann \& Wolf, 2006; Preuß et al., 2009). Participants in the $\mathrm{MoL}$ are instructed to use emotions and other sensory impressions when creating their mental image. Therefore, cortisol would be an interesting marker to investigate during the MoL task.

Besides the neurobiological substrates involved in the memory process, it is still unclear which physiological structures and signal pathways are affected by acute exercise. A recent review by Loprinzi et al (2018a) has described various reaction chains that can explain exercise-induced effects on LTP. We can only speculate about the underlying processes in the current study. One of them may involve action potentials induced by the contraction of the muscle spindles, which are transmitted via nerve pathways until the locus coeruleus (LC) is activated, affecting the hippocampus and prefrontal cortex. Future studies should be aware of body structures involved in the memory process (e.g. the vagus nerve, the LC, the NTS). We suggest to systematically evaluate how physical exercise may affect them.

\section{Conclusion}

In conclusion, the current study used a within-subjects design to investigate the influence of acute resistance exercise (fast and slow squats) on a memory task. Participants were sport students who had been instructed in a memory strategy to encode word lists. Contrary to our predictions, performing squats led to decreases instead of increases in performance, compared to encoding while sitting. Differences in memory performance between the three conditions decreased with increasing practice of the motor tasks. We conclude that the current study created a dual-task situation, leading to costs. These costs can be alleviated with increasing practice of the motor task, and with increasing levels of experience in the temporal coupling of the MoL stimuli and the squats.

Acknowledgements The authors would like to thank Margo Schleuter and David Jenner for help with data entry, and Janine Vieweg, Christian Kaczmarek and Fabian Pelzer for helpful discussions. We would also like to thank our participants for taking part in the study.

Author contributions GA and SS developed the study design and collected the data. GA conducted the literature review. TB analyzed and interpreted the data with contributions from GA. GA led the drafting of the manuscript with contributions from SS and TB.

Funding Open Access funding enabled and organized by Projekt DEAL. This work was supported by Saarland University.

Data availability Data will be made available on request.

Code availability Not applicable.

\section{Declarations}

Conflict of interest The authors declare no conflict of interest.

Ethical approval All procedures performed in studies involving human participants were in accordance with the ethical standards of the institutional and/or national research committee and with the 1964 Helsinki declaration and its later amendments or comparable ethical standards. The study was approved by the Ethics committee of Saarland University.

Informed consent Informed consent was obtained from all individual participants included in the study.

Open Access This article is licensed under a Creative Commons Attribution 4.0 International License, which permits use, sharing, adaptation, distribution and reproduction in any medium or format, as long as you give appropriate credit to the original author(s) and the source, provide a link to the Creative Commons licence, and indicate if changes were made. The images or other third party material in this article are included in the article's Creative Commons licence, unless indicated otherwise in a credit line to the material. If material is not included in the article's Creative Commons licence and your intended use is not permitted by statutory regulation or exceeds the permitted use, you will need to obtain permission directly from the copyright holder. To view a copy of this licence, visit http://creativecommons.org/licenses/by/4.0/.

\section{References}

Adams, J. (1971). A closed-loop theory of motor learning. Journal of Motor Behavior, 3, 111-149. https://doi.org/10.1080/00222895. 1971.10734898 
Amico, G., \& Schaefer, S. (2019). No evidence for performance improvements in episodic memory due to fidgeting, doodling or a "neuro-enhancing" drink. Journal of Cognitive Enhancement. https://doi.org/10.1007/s41465-019-00124-9

Amico, G., \& Schaefer, S. (2020). Running during encoding improves word learning for children. Frontiers in Psychology, 11(684). https://doi.org/10.3389/fpsyg.2020.00684

Audiffren, M., Tomporowski, P. D., \& Zagrodnik, J. (2008). Acute aerobic exercise and information processing: Energizing motor processes during a choice reaction time task. Acta Psychologica, 129(3), 410-419. https://doi.org/10.1016/j.actpsy.2008.09.006

Bates, D., Mächler, M., Bolker, B., \& Walker, S. (2015). Fitting linear mixed-effects models using lme4. ArXiv e-prints, arXiv:1406https://doi.org/10.18637/jss.v067.i01

Brehmer, Y., Stoll, G., Bergner, S., Benoit, R., von Oertzen, T., \& Lindenberger, U. (2004). Selection of unambiguous visual words appropriate for children in age-comparable memory experiments: Results of a pilot study. Available: http://psydok.sulb.uni-saarl and.de/volltexte/2004/189/.

Buchanan, T. W., \& Lovallo, W. R. (2001). Enhanced memory for emotional material following stress-level cortisol treatment in humans. Psychoneuroendocrinology, 26(3), 307-317.

Cahill, L., \& Alkire, M. T. (2003). Epinephrine enhancement of human memory consolidation: Interaction with arousal at encoding. Neurobiology of Learning and Memory, 79(2), 194-198. https://doi. org/10.1016/S1074-7427(02)00036-9

Cassilhas, R. C., Lee, K. S., Fernandes, J., Oliveira, M. G. M., Tufik, S., Meeusen, R., \& De Mello, M. T. (2012). Spatial memory is improved by aerobic and resistance exercise through divergent molecular mechanisms. Neuroscience, 202, 309-317. https://doi. org/10.1016/j.neuroscience.2011.11.029

Chang, Y.-K., \& Etnier, J. L. (2009a). Effects of an acute bout of localized resistance exercise on cognitive performance in middle-aged adults: A randomized controlled trial study. Psychology of Sport and Exercise, 10, 19-24. https://doi.org/10.1016/j.psychsport. 2008.05.004

Chang, Y.-K., \& Etnier, J. L. (2009b). Exploring the dose-response relationship between resistance exercise intensity and cognitive function. Journal of Sport and Exercise Psychology, 31(5), 640 656. https://doi.org/10.1123/jsep.31.5.640

Chang, Y.-K., Pan, C.-Y., Chen, F.-T., Tsai, C.-L., \& Huang, C.-C. (2012a). Effect of resistance-exercise training on cognitive function in healthy older adults: A review. Journal of Aging and Physical Activity, 20(4), 497-517. https://doi.org/10.1123/japa.20.4.497

Chang, Y. K., Labban, J. D., Gapin, J. I., \& Etnier, J. L. (2012b). The effects of acute exercise on cognitive performance: A meta-analysis. Brain Research, 1453, 87-101. https://doi.org/10.1016/j.brain res.2012.02.068

Coles, K., \& Tomporowski, P. D. (2008). Effects of acute exercise on executive processing, short-term and long-term memory. Journal of Sports Sciences, 26, 333-344. https://doi.org/10.1080/02640 410701591417

Cooper, C. J. (1973). Anatomical and physiological mechanisms of arousal, with special reference to the effects of exercise. Ergonomics, 16(5), 601-609. https://doi.org/10.1080/00140137308924551

Cuttler, C., Connolly, C. P., LaFrance, E. M., \& Lowry, T. M. (2018). Resist forgetting: Effects of aerobic and resistance exercise on prospective and retrospective memory. Sport, Exercise, and Performance Psychology, 7(2), 205-217. https://doi.org/10.1037/ spy0000112

Díaz-Mataix, L., Scorza, M. C., Bortolozzi, A., Toth, M., Celada, P., \& Artigas, F. (2005). Involvement of 5-HT1A receptors in prefrontal cortex in the modulation of dopaminergic activity: Role in atypical antipsychotic action. The Journal of Neuroscience,
25(47), 10831-10843. https://doi.org/10.1523/JNEUROSCI.299905.2005

Etnier, J., Labban, J. D., Piepmeier, A., Davis, M. E., \& Henning, D. A. (2014). Effects of an acute bout of exercise on memory in 6th grade children. Pediatric Exercise Science, 26(3), 250-258. https://doi.org/10.1123/pes.2013-0141

Finlay, J. M., Zigmond, M. J., \& Abercrombie, E. D. (1995). Increased dopamine and norepinephrine release in medial prefrontal cortex induced by acute and chronic stress: Effects of diazepam. Neuroscience, 64(3), 619-628. https://doi.org/10.1016/0306-4522(94) 00331-x

Fitts, M., \& Posner, M. I. (1967). Human performance. Brooks/Cole.

Frey, U., \& Morris, R. G. (1997). Synaptic tagging and long-term potentiation. Nature, 385(6616), 533-536. https://doi.org/10. 1038/385533a0

Frith, E., Sng, E., \& Loprinzi, P. D. (2017). Randomized controlled trial evaluating the temporal effects of high-intensity exercise on learning, short-term and long-term memory, and prospective memory. European Journal of Neuroscience, 46(10), 2557-2564. https://doi.org/10.1111/ejn.13719

Gentile, A. M. (1972). A working model of skill acquisition with application to teaching. Quest, 17, 3-23. https://doi.org/10.1080/00336 297.1972.10519717

Hansen, N., \& Manahan-Vaughan, D. (2015). Hippocampal long-term potentiation that is elicited by perforant path stimulation or that occurs in conjunction with spatial learning is tightly controlled by beta-adrenoreceptors and the locus coeruleus. Hippocampus, 25(11), 1285-1298. https://doi.org/10.1002/hipo.22436

Harveson, A. T., Hannon, J. C., Brusseau, T. A., Podlog, L., Papadopoulos, C., Durrant, L. H., Hall, M. S., \& Kang, K.-D. (2016). Acute effects of 30 Minutes resistance and aerobic exercise on cognition in a high school sample. Research Quarterly for Exercise and Sport, 87(2), 214-220. https://doi.org/10.1080/02701367. 2016.1146943

Hillman, C., Kamijo, K., \& Scudder, M. R. (2011). A review of chronic and acute physical activity participation on neuroelectric measures of brain health and cognition during childhood. Preventive Medicine, 52, 21-28. https://doi.org/10.1016/j.ypmed.2011.01.024

Hötting, K., Schickert, N., Kaiser, J., Röder, B., \& Schmidt-Kassow, M. (2016). The effects of acute physical exercise on memory, peripheral BDNF, and cortisol in young adults. Neural Plasticity, 1-12. https://doi.org/10.1155/2016/6860573

Kliegl, R., Smith, J., \& Baltes, P. (1990). On the locus and process of magnification of age differences during mnemonic training. Developmental Psychology, 26, 894-904. https://doi.org/10.1037/ 0012-1649.26.6.894

Kliegl, R., Smith, J., \& Baltes, P. B. (1989). Testing-the-limits and the study of adult age differences in cognitive plasticity of a mnemonic skill. Developmental Psychology, 25, 247-256. https://doi. org/10.1037/0012-1649.25.2.247

Knaepen, K., Goekint, M., Heyman, E. M., \& Meeusen, R. (2010). Neuroplasticity - Exercise-induced response of peripheral brainderived neurotrophic factor: A systematic review of experimental studies in human subjects. Sports Medicine, 40, 765-801. https:// doi.org/10.2165/11534530-000000000-00000

Kuhlmann, S., \& Wolf, O. T. (2006). Arousal and cortisol interact in modulating memory consolidation in healthy young men. Behavioral Neuroscience, 120(1), 217-223. https://doi.org/10.1037/ 0735-7044.120.1.217

Kuznetsova, A., Brockhoff, P., \& Christensen, R. (2015). LmerTest: Tests in linear mixed effects models. $R$ Package Version, 2. https:// doi.org/10.18637/jss.v082.i13

Labban, J. D., \& Etnier, J. L. (2011). Effects of acute exercise on longterm memory. Research Quarterly for Exercise and Sport, 82(4), 712-721. https://doi.org/10.1080/02701367.2011.10599808 
Landrigan, J.-F., Bell, T., Crowe, M., Clay, O. J., \& Mirman, D. (2019). Lifting cognition: A meta-analysis of effects of resistance exercise on cognition. Psychological Research Psychologische Forschung. https://doi.org/10.1007/s00426-019-01145-x

Li, K. Z. H., Lindenberger, U., Freund, A. M., \& Baltes, P. B. (2001). Walking while memorizing: Age-related differences in compensatory behavior. Psychological Science, 12, 230-237. https://doi. org/10.1111/1467-9280.00341

Li, Q., Rothkegel, M., Xiao, Z. C., Abraham, W. C., Korte, M., \& Sajikumar, S. (2014). Making synapses strong: Metaplasticity prolongs associativity of long-term memory by switching synaptic tag mechanisms. Cerebral Cortex, 24(2), 353-363. https://doi.org/ 10.1093/cercor/bhs315

Lindenberger, U., Marsiske, M., \& Baltes, P. B. (2000). Memorizing while walking: Increase in dual-task costs from young adulthood to old age. Psychology and Aging, 15, 417-436. https://doi.org/ 10.1037/0882-7974.15.3.417

Loprinzi, P. D., Blough, J., Crawford, L., Ryu, S., Zou, L., \& Li, H. (2019a). The temporal effects of acute exercise on episodic memory function: Systematic review with meta-analysis. Brain Sciences, 9(4). https://doi.org/10.3390/brainsci9040087

Loprinzi, P. D., Edwards, M. K., \& Frith, E. (2017). Potential avenues for exercise to activate episodic memory-related pathways: A narrative review. European Journal of Neuroscience, 46(5), 2067-2077. https://doi.org/10.1111/ejn.13644

Loprinzi, P. D., \& Frith, E. (2019). A brief primer on the mediational role of BDNF in the exercise-memory link. Clinical Physiology and Functional Imaging, 39(1), 9-14. https://doi.org/10.1111/cpf. 12522

Loprinzi, P. D., Frith, E., \& Edwards, M. K. (2018a). Resistance exercise and episodic memory function: A systematic review. Clinical Physiology and Functional Imaging. https://doi.org/10.1111/cpf. 12507

Loprinzi, P. D., Koehler, L., Frith, E., Ponce, P., Delancey, D., Joyner, C., Ashpole, N. M., Zou, L., \& Li, H. (2019b). Acute exercise, psychological stress induction, and episodic memory. American Journal of Health Behavior, 43(6), 1016-1029. https://doi.org/ 10.5993/AJHB.43.6.1

Loprinzi, P. D., Ponce, P., \& Frith, E. (2018b). Hypothesized mechanisms through which acute exercise influences episodic memory. Physiology International, 105(4), 285-297. https://doi.org/10. $1556 / 2060.105 .2018 .4 .28$

McMorris, T. (2016). Exercise-cognition interactions: Neuroscience Perspectives: Elsevier.

McMorris, T., \& Graydon, J. (2000). The effect of incremental exercise on cognitive performance. International Journal of Sport Psychology, 31, 66-81.

McMorris, T., \& Hale, B. J. (2012). Differential effects of differing intensities of acute exercise on speed and accuracy of cognition: A meta-analytical investigation. Brain and Cognition, 80, 338-351. https://doi.org/10.1016/j.bandc.2012.09.001

Meeusen, R., \& De Meirleir, K. (1995). Exercise and brain neurotransmission. Sports Medicine, 20(3), 160-188. https://doi.org/ 10.2165/00007256-199520030-00004

Meinel, K. (1960). Bewegungslehre. Versuch einer Theorie der sportlichen Bewegung unter pädagogischem Aspekt. Verlag Volk und Wissen.

Pesce, C., Crova, C., Cereatti, L., Casella, R., \& Bellucci, M. (2009). Physical activity and mental performance in preadolescents: Effects of acute exercise on free-recall memory. Mental Health and Physical Activity, 2, 16-22. https://doi.org/10.1016/j.mhpa. 2009.02.001

Physical Activity Guidelines Advisory Committee. (2008). Physical activity guidelines advisory committee report. Department of Health and Human Services.
Preuß, D., Schoofs, D., \& Wolf, O. T. (2009). Associations between endogenous cortisol levels and emotional memory in young women: Influence of encoding instructions. Stress, 12(5), 379387. https://doi.org/10.1080/10253890802524592

Pyke, W., Ifram, F., Coventry, L., Sung, Y., Champion, I., \& Javadi, A.-H. (2020). The effects of different protocols of physical exercise and rest on long-term memory. Neurobiology of Learning and Memory, 167, 107128. https://doi.org/10.1016/j.nlm.2019.107128

Raudenbush, S. W., \& Bryk, A. S. (2002). Hierarchical linear models: Applications and data analysis methods (Vol. 1). Sage Publications.

Roig, M., Nordbrandt, S., Geertsen, S. S., \& B., N. J. . (2013). The effects of cardiovascular exercise on human memory: A review with meta-analysis. Neuroscience and Biobehavioral Reviews, 37, 1645-1666. https://doi.org/10.1016/j.neubiorev.2013.06.012

Roig, M., Skriver, K., Lundbye-Jensen, J., Kiens, B., \& Nielsen, J. B. (2012). A single bout of exercise improves motor memory. PLoS ONE, 7, e44594.

Roig, M., Thomas, A. G., Mang, C. S., Snow, N. J., Ostadan, F., Boyd, L. A., \& Lundbye-Jensen, J. (2016). Time-dependent effects of cardiovascular exercise on memory. Exercise and Sport Science Reviews, 44, 81-88. https://doi.org/10.1249/JES.0000000000 000078

Sajikumar, S., \& Frey, J. U. (2004). Late-associativity, synaptic tagging, and the role of dopamine during LTP and LTD. Neurobiology of Learning and Memory, 82(1), 12-25. https://doi.org/10. 1016/j.nlm.2004.03.003

Schaefer, S. (2014). The ecological approach to cognitive-motor dualtasking: Findings on the effects of expertise and age. Frontiers in Psychology, 5, 1-9. https://doi.org/10.3389/fpsyg.2014.01167

Schaefer, S., Krampe, R. T., Lindenberger, U., \& Baltes, P. B. (2008). Age differences between children and young adults in the dynamics of dual-task prioritization: Body (balance) versus mind (memory). Developmental Psychology, 44, 747-757. https://doi.org/10. 1037/0012-1649.44.3.747

Schmidt-Kassow, M., Deusser, M., Thiel, C., Otterbein, S., Montag, C., Reuter, M., Banzer, W., \& Kaiser, J. (2013). Physical exercise during encoding improves vocabulary learning in young female adults: A neuroendocrinological study. PLoS ONE, 8, e64172. https://doi.org/10.1371/journal.pone.0064172

Schmidt-Kassow, M., Kulka, A., Gunter, T. C., Rothermich, K., \& Kotz, S. A. (2010). Exercising during learning improves vocabulary acquisition: Behavioral and ERP evidence. Neuroscience Letters, 482(1), 40-44. https://doi.org/10.1016/j.neulet.2010.06.089

Schmidt-Kassow, M., Zink, N., Mock, J., Thiel, C., Vogt, L., Abel, C., \& Kaiser, J. (2014). Treadmill walking during vocabulary encoding improves verbal long-term memory. Behavioral and Brain Functions, 10, 24. https://doi.org/10.1186/1744-9081-10-24

Schramke, C. J., \& Bauer, R. M. (1997). State-dependent learning in older and younger adults. Psychology and Aging, 12(2), 255-262. https://doi.org/10.1037//0882-7974.12.2.255

Segal, S. K., Cotman, C. W., \& Cahill, L. F. (2012). Exercise-induced noradrenergic activation enhances memory consolidation in both normal aging and patients with amnestic mild cognitive impairment. Journal of Alzheimer's Disease : JAD, 32(4), 1011-1018. https://doi.org/10.3233/JAD-2012-121078

Singh, A. S., Saliasi, E., van den Berg, V., Uijtdewilligen, L., de Groot, R. H. M., Jolles, J., . . Chinapaw, M. J. M. (2018). Effects of physical activity interventions on cognitive and academic performance in children and adolescents: a novel combination of a systematic review and recommendations from an expert panel. British Journal of Sports Medicine, 53(10), 640-647. https://doi. org/10.1136/bjsports-2017-098136

Skriver, K., Roig, M., Lundbye-Jensen, J., Pingel, J., Helge, J. W., Kiens, B., \& Nielsen, J. B. (2014). Acute exercise improves motor 
memory: Exploring potential biomarkers. Neurobiology of Learning and Memory, 116, 46-58. https://doi.org/10.1016/j.nlm.2014. 08.004

Snijders, T., \& Bosker, R. (2004). Multilevel Analysis. Sage.

Sng, E., Frith, E., \& Loprinzi, P. D. (2018). Experimental effects of acute exercise on episodic memory acquisition: Decomposition of multi-trial gains and losses. Physiology and Behavior, 186, 82-84. https://doi.org/10.1016/j.physbeh.2018.01.014

Tomporowski, P. D. (2003). Effects of acute bouts of exercise on cognition. Acta Psychologica, 112, 297-324. https://doi.org/10.1016/ S0001-6918(02)00134-8

Tomporowski, P. D., Davis, C. L., Miller, P. H., \& Naglieri, J. A. (2008). Exercise and children's intelligence, cognition, and academic achievement. Educational Psychology Reviews, 20, 111131. https://doi.org/10.1007/s10648-007-9057-0

Verburgh, L., Königs, M., Scherder, E. J. A., \& Oosterlaan, J. (2014). Physical exercise and executive functions in preadolescent children, adolescents and young adults: A meta-analysis. British Journal of Sports Medicine, 48(12), 973-979. https://doi.org/10.1136/ bjsports-2012-091441

Wechsler, D. (1981). Wechsler Adult Intelligence Scale - Revised $(W A I S-R)$. New York: Psychological Corporation.
Weinberg, L., Hasni, A., Shinohara, M., \& Duarte, A. (2014). A single bout of resistance exercise can enhance episodic memory performance. Acta Psychologica (amst), 153, 13-19. https://doi.org/10. 1016/j.actpsy.2014.06.01q

Wickens, C. D. (1991). Processing resources and attention. In D. Damos (Ed.), Multiple-task performance (pp. 3-34). Taylor-Francis.

Wilke, J., Giesche, F., Klier, K., Vogt, L., Herrmann, E., \& Banzer, W. (2019). Acute effects of resistance exercise on cognitive function in healthy adults: A systematic review with multilevel meta-analysis. Sports Medicine (auckland, N. Z.), 49(6), 905-916. https:// doi.org/10.1007/s40279-019-01085-x

Winter, B., Breitenstein, C., Mooren, F. C., Voelker, K., Fobker, M., Lechtermann, A., . . Knecht, S. (2007). High impact running improves learning. Neurobiology of Learning and Memory, 87, 597-609. https://doi.org/10.1016/j.nlm.2006.11.003

Publisher's note Springer Nature remains neutral with regard to jurisdictional claims in published maps and institutional affiliations. 